\title{
Editorial
}

\section{Health Sector Response to HIV in the SAARC Region}

For nearly 15 years, the Millennium Development Goals (MDGs) have been a guiding force on many issues affecting the lives of children, young people and their families. Much progress has been made in getting more needy people into treatment coverage, reducing new HIV infection and AIDS related death in SAARC Region.

The Health Sector of Member States of SAARC Region has been playing a pivotal role in the national response to HIV. Member States have enormous opportunities, in the context of universal health coverage, to further scale up their response to HIV with innovative service delivery models, including mass coverage and decentralization of HIV testing and treatment services. Some Member States also initiated integration of HIV services with maternal and child health services, family planning and tuberculosis control programme.

At the end of 2013, the SAARC Region have estimated 2.20 million people living with HIVIAIDS and more people than ever are receiving live saving antiretroviral treatment. Optimizing the outcome of HIV prevention, diagnosis treatment and care, Member States have focused on strengthening the health system too. The effective integration of health services is to ensure availability, access, affordability and quality, including strengthening of human resources, providing innovative approaches to service delivery and utilizing laboratory support. Also creating a supportive and enabling environment to ensure equitable access to HIV services and reducing HIV-related stigma and discrimination.

The significant results achieved over the last ten years can be largely attributed to the scientific, programmatic and governance innovations of the AIDS response. While the significant achievements in the AIDS response are encouraging, the epidemic is far from over and entrenched challenges still remain. Globally, HIV is the sixth leading cause of adult mortality and the largest killer among women aged 15-49 years.

Health systems in many low- and middle-income countries need strengthening, as they are still unable to provide necessary HIV-related health services to the general population. Evidence suggests that an AIDS investment has improved not only the delivery of HIV-related services but strengthened health systems in many countries. At the same time, much more still need to be done to ensure this integrated and synergistic activities with health services particularly around sexual and reproductive health and maternal and child health.

MDGs era coming to an end this year, 2015, the Region has a historic opportunity to set a course for the next era of human development that is transformational for both people and planet. 